\title{
Incidental Squamous Cell Carcinoma in an Epidermal Inclusion Cyst: A Case Report and Review of the Literature
}

\author{
Ethan Frank $^{\mathrm{a}} \quad$ David Macias $^{\mathrm{a}} \quad$ Brian Hondorp $^{\mathrm{b}} \quad$ Justin Kerstetter $^{\mathrm{c}}$ \\ Jared C. Inman ${ }^{b}$ \\ aLoma Linda University School of Medicine, Loma Linda, CA, USA; ${ }^{b}$ Department of \\ Otolaryngology - Head and Neck Surgery, Loma Linda University, Loma Linda, CA, USA; \\ 'Department of Pathology and Laboratory Medicine, Loma Linda University, Loma \\ Linda, CA, USA
}

\section{Keywords}

Epidermal cyst · Inclusion cyst · Cutaneous cyst · Squamous cell carcinoma · Malignant transformation

\begin{abstract}
Epidermal inclusion cysts are common lesions that rarely develop into squamous cell carcinoma (SCC). Neoplastic change in these cysts can be associated with prominent symptoms such as pain, rapid growth, or ulceration. This study describes the case of a 64-year-old woman with a 4-year history of a largely asymptomatic neck mass, which after routine excision was found to be an epidermal inclusion cyst harboring well-differentiated SCC. The diagnosis was made incidentally after routine cyst bisection and hematoxylin and eosin staining. Given the potential for variable presentation and low cost of hematoxylin and eosin analysis, we recommend a low threshold for a comprehensive pathological search for malignancy in excised cysts when appropriate.


Frank et al.: Incidental Squamous Cell Carcinoma in an Epidermal Inclusion Cyst: A Case

Report and Review of the Literature

\section{Introduction}

Cutaneous epidermal inclusion cysts are frequently encountered and histologically benign subcutaneous lesions that arise most often along embryonic fusion lines as a result of invagination of keratinized squamous epithelium. Although epidermal inclusion cysts account for approximately 85-95\% of all excised cysts, malignant transformation is exceedingly rare. We report a case of squamous cell carcinoma (SCC) arising from an epidermal inclusion cyst of the neck in a 64-year-old woman.

\section{Case Report}

A 64-year old female presented with a 4-year history of a nontender neck mass that had slowly increased in size over the past year. The patient reported no changes in voice, swallowing, or breathing. Physical examination revealed a mobile subcutaneous mass in the left lower neck that was approximately $11 \mathrm{~mm}$ in diameter. There were no overlying skin changes. Ultrasonography revealed a homogenous, hypoechoic, and well-circumscribed cystic mass within the subcutaneous fat of the neck suggestive of a benign lesion. Routine excision was performed and the cyst was found to be free from surrounding structures and nonfibrotic. Pathological analysis included bisection of the cyst with hematoxylin and eosin (HE) staining, revealing an epidermal inclusion cyst with an incidental finding of focal nests of atypia and a focus of well-differentiated SCC (Fig. 1, 2, 3). Total cyst size was $2.1 \mathrm{~cm}$ with the SCC component occupying $4 \mathrm{~mm}$. The surgical margins were free of neoplastic involvement. At the 1-year follow-up, the patient was well with no evidence of recurrence.

\section{Discussion}

While cutaneous epidermal inclusion cysts are a common lesion, the development of SCC from the wall of the cyst is an infrequent occurrence. The incidence of SCC developing from an epidermal inclusion cyst has been estimated to range from 0.011 to $0.045 \%$ [1]. After a review of the literature, we found 41 well-documented cases of SCC arising from cutaneous epidermal inclusion cysts [1-13]. Our findings are summarized in Table 1 and Table 2, which shows an increase in the number of reported cases of SCC arising from epidermal inclusion cysts since 2010. If this phenomenon is due to a rising incidence of malignant transformation or increased reporting is difficult to ascertain.

According to Table 1 and Table 2, the mean age of diagnosis of SCC arising from an epidermal inclusion cysts was 61.8 years (range, 28-96 years) with men more often affected (69.0\%). The most common site of occurrence was the head and neck (54.8\%). Cyst size varied significantly with a mean cyst diameter of $5.0 \mathrm{~cm}$ (range, $0.7-20 \mathrm{~cm}$ ). The mean duration of cyst presence was 92.6 months (range, 0.5-480 months), whereas the mean time to diagnosis once the cyst became symptomatic was 7.3 months (range, $0.1-24$ months). The most common presenting symptoms were pain (24.2\%), rapid cyst enlargement $(48.6 \%)$, and overlying skin changes such as erythema, ulceration, or drainage (38.2\%). Twenty-five percent of cases had failed antibiotic therapy prior to excision of the cyst. 
The specific mechanism behind malignant transformation of epidermal inclusion cysts to SCC is uncertain. Some have proposed that chronic cyst irritation in the form of chronic inflammation or infection can trigger malignant change as it is a recognized mechanism of cancer development at other body sites. Other proposed mechanisms of malignant transformation of epidermal inclusion cysts include direct trauma, human papillomavirus, immunosuppression, and actinic damage, although little research exists to support these theories $[1,12]$.

Due to the rare nature of the disease, the clinical course and ideal method of management is not well established. In our review of the literature, we found several warning symptoms that would increase suspicion of malignant degeneration of an epidermal inclusion cyst. These were cysts presenting with pain, rapid growth, or overlying skin changes such as ulceration or persistent drainage. However, these symptoms can often indicate cyst infection and reports detailing specific and reliable symptomatic patterns to suggest malignancy are lacking. The index of suspicion of malignant transformation should be raised whenever suspected infected cysts fail to respond to medical management such as antibiotics. Furthermore, while prominent symptoms such as rapid enlargement may potentially be sensitive indicators of malignant transformation, the absence of such symptoms cannot exclude the diagnosis as multiple cases reported gradual growth over a long period of time or no growth at all.

Although malignant transformation of an epidermal inclusion cyst is rare, cysts that are concerning should have a low threshold for excision and thorough histopathological examination. The primary treatment for a neoplastic epidermal inclusion cyst is wide excision with adequate margins of $4 \mathrm{~mm}$ for all lesions and at least $6 \mathrm{~mm}$ for higher-risk cysts [3]. Given the low probability of SCC in an epidermal inclusion cyst, a significant number of these malignancies are detected incidentally during histopathological analysis following excision of a presumed benign cyst. Unfortunately, there is no standardized pathological examination for epidermal inclusion cysts. A simple bisection of a cyst that is assumed to be benign may miss malignant degeneration in the lateral walls of the cyst, yet it is likely neither reasonable nor cost-effective to section every epidermal inclusion cyst as if it were neoplastic. Risk stratification with attention to cases suspicious for malignancy must guide both the surgeon and pathologist. The most cost-effective way to section these cysts is unclear, and the surgeon may need to direct the specimen sectioning when neoplastic change is suspected, such as when concerning symptoms are present, the cyst is fixed to surrounding structures, or fibrosis is present.

The possibility of more thorough histopathological analysis of epidermal inclusion cysts with suspected malignant degeneration must be balanced with the added cost burden. A cost analysis at our institution was performed for standard staining and microscopic examination. Taking into account the staining materials, labor, instrumentation, and company cost allocation, we found the price of HE staining and analysis to cost between 0.50 and 1.00 USD per slide. In light of the low cost of pathological analysis using HE, we suggest a low threshold for thorough sectioning of cysts when malignant transformation is suspected. However, given the rarity of the disease, standardizing stringent sectioning techniques to all epidermal inclusion cysts is probably unwarranted. Further research is likely impractical due to a low incidence of malignant transformation within epidermal inclusion cysts. 
SCC arising in cutaneous epidermal inclusion cysts is a rare occurrence in the medical literature. The low incidence, along with variance in description of features and symptoms of malignant epidermal inclusion cysts precludes the formation of specific guidelines for evaluation and management. Detailed pathological analysis should be considered when the suspicion of malignant degeneration is high, or when the cyst presents with pain, rapid growth, overlying skin change, or if a presumably infected cyst does not respond to medical management.

\section{Statement of Ethics}

This project was approved by our institution's IRB.

\section{Disclosure Statement}

The authors have no conflicts of interest to disclose.

\section{References}

1 Veenstra JJ, Choudhry S, Krajenta RJ, Eide MJ: Squamous cell carcinoma originating from cutaneous cysts: the Henry Ford experience and review of literature. J Dermatolog Treat 2016;27:95-98.

2 Pusiol T, Zorzi MG, Piscioli F: Squamous cell carcinoma arising in epidermal and human papillomavirus associated cysts: report of three cases. Pathologica 2010;102:88-92.

3 Shabbir A, Loss L, Bogner P, Zeitouni NC: Squamous cell carcinoma developing from an epidermoid cyst of the ear. Dermatol Surg 2011;37:700-703.

4 Kshirsagar AY, Sulhyan SR, Deshpande S, Jagtap S: Malignant change in an epidermal cyst over gluteal region. J Cutan Aesthet Surg 2011;4:48-50.

5 Milbratz GH, Borges FP, Cintra MB, Silva GE, Velasco e Cruz AA: Orbital invasion by squamous cell carcinoma arising in multiple epidermoid cysts. Ophthal Plast Reconstr Surg 2012;28:144-145.

6 Morritt AN, Tiffin N, Brotherston TM: Squamous cell carcinoma arising in epidermoid cysts: report of four cases and review of literature. J Plast Reconstr Aesthet Surg 2012;65:1267-1269.

7 Skroza N, Proietti I, Tolino E, et al: Isotretinoin for the treatment of squamous cell carcinoma arising on an epidermoid cyst. Dermatol Ther 2014;27:94-96.

8 Sridevi HB, Shariff MH, Pushpalatha PK: Squamous cell carcinoma arising in an epidermal cyst. Indian J Cancer 2015;52:335-336.

9 Sakamoto A, Shiba E, Hisaoka M: Squamous cell carcinoma arising from an epidermal cyst in the thumb. Int J Surg Case Rep 2015;11:37-39.

10 Suhani, Aggarwal L, Meen K, Ali S, Thomas S: Squamous cell carcinoma arising in epidermal inclusion cyst of breast: a diagnostic dilemma. Breast Dis 2015;35:25-27.

11 Lee JW, Shin JY, Roh SG, Lee NH, Yang KM: Squamous cell carcinoma arising from an epidermal inclusion cyst. Arch Plast Surg 2016;43:112-114. Sze S, Richmond I, Bickers A, Saha A: Squamous cell carcinoma arising from a vulval epidermal cyst. J Obstet Gynaecol Res 2016;42:1623-1626.

13 Mohan C, Srivastava A, Agrawal R, Bhardwaj P: A rare case of epidermoid cyst in neck. Int J Adv Integr Med Sci 2016;1:15-17. 


\section{Case Reports in Dermatology}

Case Rep Dermatol 2018;10:61-68

DOI: $10.1159 / 000487794$

(c) 2018 The Author(s). Published by S. Karger AG, Basel www.karger.com/cde

Frank et al.: Incidental Squamous Cell Carcinoma in an Epidermal Inclusion Cyst: A Case

Report and Review of the Literature

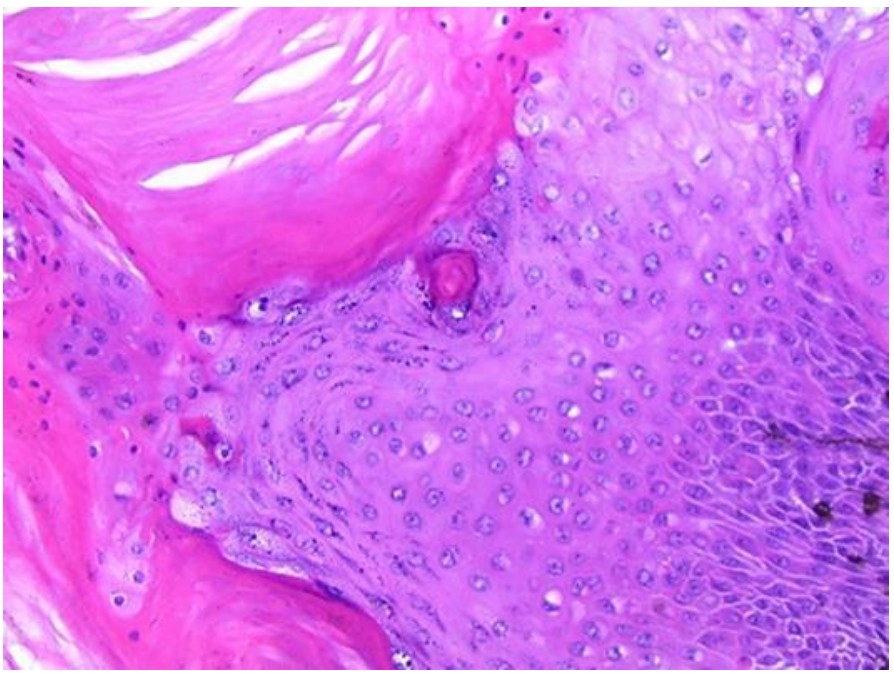

Fig. 1. Low-power photomicrograph depicting granular layer with associated keratinization consistent with epidermoid-infundibular cyst. HE staining.

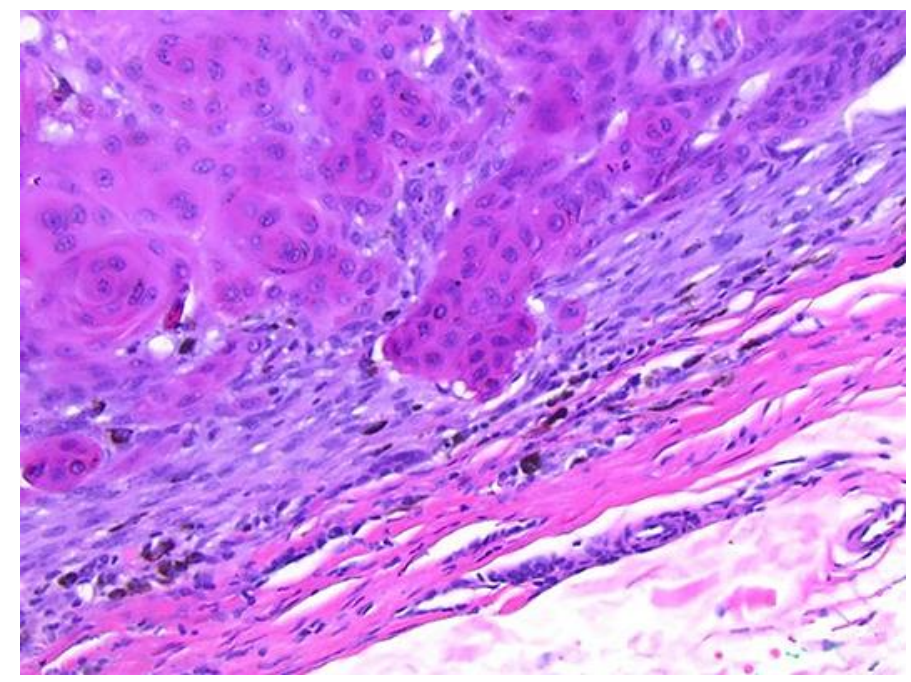

Fig. 2. Medium-power view showing atypical squamous epithelium with focal infiltration and associated stromal response. HE staining. 


\section{Case Reports in Dermatology}

Case Rep Dermatol 2018;10:61-68 DOI: $10.1159 / 000487794$

(c) 2018 The Author(s). Published by S. Karger AG, Basel www.karger.com/cde

Frank et al.: Incidental Squamous Cell Carcinoma in an Epidermal Inclusion Cyst: A Case Report and Review of the Literature

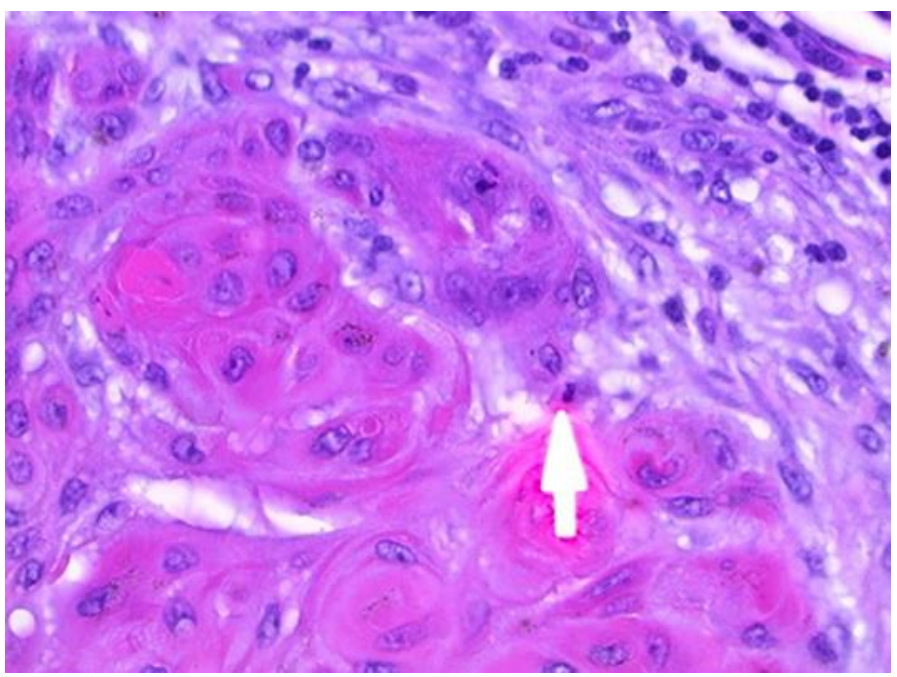

Fig. 3. Medium-power view demonstrating keratinizing tumor cells with mitotic figure. HE staining. 
Table 1. Literature review - cases of SCC arising from cutaneous epidermal inclusions cysts

\begin{tabular}{|c|c|c|c|c|c|c|c|c|c|c|}
\hline Case & $\begin{array}{l}\text { Authors } \\
\text { [Ref.] }\end{array}$ & Year & $\begin{array}{l}\text { Age, } \\
\text { sex }\end{array}$ & Body site & $\begin{array}{l}\text { Size, } \\
\mathrm{cm}^{1}\end{array}$ & $\begin{array}{l}\text { Duration, } \\
\text { months }^{2}\end{array}$ & $\begin{array}{l}\text { New s/s, } \\
\text { months }\end{array}$ & Treatment & Outcome & $\begin{array}{l}\text { Sectioning } \\
\text { technique }^{4}\end{array}$ \\
\hline \multirow[t]{25}{*}{$1-25$} & \multirow{25}{*}{$\begin{array}{l}\text { Veenstra } \\
\text { et al. [1] }\end{array}$} & 1976 & $52, \mathrm{M}$ & R ear & NS & 2 & NS & Excision & No recurrence & NS \\
\hline & & 1980 & $68, \mathrm{M}$ & R preauricular & 3 & 4 & NS & $\begin{array}{l}\text { Excision, } \\
\text { parotidectomy }\end{array}$ & No recurrence & NS \\
\hline & & 1981 & $34, \mathrm{M}$ & L retroauricular & 8 & NS & 24 & Excision, RT & No recurrence & NS \\
\hline & & 1982 & $58, \mathrm{M}$ & R ear & 2.5 & 132 & NS & Excision & No recurrence & NS \\
\hline & & 1987 & $59, \mathrm{~F}$ & L thigh & 7 & 3 & 3 & $\begin{array}{l}\text { Excision, inguinal } \\
\text { dissection }\end{array}$ & $\begin{array}{l}\text { Inguinal mets, } \\
\text { death at } 6 \text { months }\end{array}$ & $\begin{array}{l}\text { "Extensive } \\
\text { sampling" }\end{array}$ \\
\hline & & 1989 & $55, \mathrm{~F}$ & L gluteal region & 10 & 6 & NS & Excision & No recurrence & NS \\
\hline & & 1994 & $32, \mathrm{M}$ & L index finger & NS & 120 & NS & Amputation, RT & No recurrence & NS \\
\hline & & 1999 & $66, \mathrm{M}$ & L retroauricular & 1.5 & 2 & 2 & Excision & No recurrence & $\begin{array}{l}\text { "Routine } \\
\text { fixing" }\end{array}$ \\
\hline & & 1999 & $92, \mathrm{~F}$ & $\mathrm{R}$ forehead & 3.5 & NS & NS & $\begin{array}{l}\text { Excision, } \\
\text { re-excision }\end{array}$ & No recurrence & NS \\
\hline & & 2000 & $57, \mathrm{M}$ & Gluteal region & 6 & 240 & NS & Excision & No recurrence & NS \\
\hline & & 2002 & $68, \mathrm{M}$ & $\mathrm{L}$ axilla & 4.5 & 24 & NS & Excision & NS & NS \\
\hline & & 2002 & $38, F$ & L buttock & 20 & 240 & NS & Excision & No recurrence & NS \\
\hline & & 2003 & $67, \mathrm{M}$ & R temple & 3 & 3 & 3 & $\begin{array}{l}\text { Excision, } \\
\text { re-excision }\end{array}$ & No recurrence & NS \\
\hline & & 2006 & $48, \mathrm{~F}$ & R abdomen & 9.2 & $>120$ & 6 & Excision, CT & $\begin{array}{l}\text { Lung mets, } \\
\text { death at } 10 \text { months }\end{array}$ & NS \\
\hline & & 2007 & $48, \mathrm{M}$ & L buttock & 5 & 336 & 3 & $\begin{array}{l}\text { Excision, inguinal } \\
\text { dissection, RT }\end{array}$ & $\begin{array}{l}\text { Mets, death at } \\
5 \text { months }\end{array}$ & NS \\
\hline & & 2007 & $74, \mathrm{M}$ & L thigh & 13 & 480 & 36 & $\begin{array}{l}\text { Excision, } \\
\text { re-excision }\end{array}$ & No recurrence & NS \\
\hline & & 2012 & $76, \mathrm{~F}$ & Perineum & 12 & 48 & NS & Excision & No recurrence & NS \\
\hline & & 2010 & $65, \mathrm{M}$ & $\mathrm{R}$ retroauricular & 2 & 2 & NS & $\begin{array}{l}\text { Excision, } \\
\text { re-excision, RT, } \\
\text { amputation }\end{array}$ & No recurrence & $\begin{array}{l}\text { "Multiple } \\
\text { serial } \\
\text { sections" }\end{array}$ \\
\hline & & 2010 & $50, \mathrm{M}$ & Head & 1.5 & 36 & NS & Excision & No recurrence & Bisection \\
\hline & & 2012 & $65, \mathrm{M}$ & L neck & 9 & 420 & 6 & Excision, RT & NS & NS \\
\hline & & 2013 & $86, \mathrm{M}$ & R scrotum & 4.1 & 276 & 0.1 & Excision & NS & NS \\
\hline & & 2013 & $63, \mathrm{M}$ & L nose & 2 & 36 & 2 & Excision & NS & NS \\
\hline & & 2016 & $46, \mathrm{~F}$ & L thigh & 2 & $>12$ & NS & $\begin{array}{l}\text { Excision, } \\
\text { re-excision }\end{array}$ & No recurrence & NS \\
\hline & & & $89, \mathrm{~F}$ & $\begin{array}{l}\text { Suprapubic } \\
\text { region }\end{array}$ & 4 & $>1$ & NS & Excision & No recurrence & NS \\
\hline & & & $61, \mathrm{M}$ & L thigh & 1.2 & $>6$ & NS & $\begin{array}{l}\text { Excision, } \\
\text { re-excision }\end{array}$ & No recurrence & NS \\
\hline
\end{tabular}


Table 2. Literature review - cases of SCC arising from cutaneous epidermal inclusions cysts (continued)

\begin{tabular}{|c|c|c|c|c|c|c|c|c|c|c|}
\hline Case & $\begin{array}{l}\text { Authors } \\
\text { [Ref.] }\end{array}$ & Year & $\begin{array}{l}\text { Age, } \\
\text { sex }\end{array}$ & Body site & $\begin{array}{l}\text { Size, } \\
\mathrm{cm}^{1}\end{array}$ & $\begin{array}{l}\text { Duration, } \\
\text { months }^{2}\end{array}$ & $\begin{array}{l}\text { New s/s, } \\
\text { months }^{3}\end{array}$ & Treatment & Outcome & $\begin{array}{l}\text { Sectioning } \\
\text { technique }^{4}\end{array}$ \\
\hline \multirow[t]{2}{*}{$26-27$} & \multirow[t]{2}{*}{$\begin{array}{l}\text { Pusiol } \\
\text { et al. [2] }\end{array}$} & \multirow[t]{2}{*}{2010} & $88, \mathrm{M}$ & $\begin{array}{l}\text { R zygomatic } \\
\text { area }\end{array}$ & 0.7 & NS & NS & Excision & No recurrence & $\begin{array}{l}\text { Serial } \\
\text { sectioning }\end{array}$ \\
\hline & & & $96, \mathrm{M}$ & R ear & 1.5 & 12 & 12 & Excision & No recurrence & $\begin{array}{l}\text { Serial } \\
\text { sectioning }\end{array}$ \\
\hline 28 & $\begin{array}{l}\text { Shabbir } \\
\text { et al. [3] }\end{array}$ & 2011 & $63, \mathrm{M}$ & L ear & 1.2 & 30 & NS & Excision & No recurrence & NS \\
\hline 29 & $\begin{array}{l}\text { Kshirsagar } \\
\text { et al. [4] }\end{array}$ & 2011 & $72, \mathrm{M}$ & L gluteal region & 12 & 120 & 0.5 & Excision & NS & $\begin{array}{l}\text { "Multiple } \\
\text { sections" }\end{array}$ \\
\hline 30 & $\begin{array}{l}\text { Milbratz } \\
\text { et al. [5] }\end{array}$ & 2012 & $60, \mathrm{M}$ & L face & 2 & 4 & 3 & $\begin{array}{l}\text { Excision, CT, } \\
\text { RT }\end{array}$ & NS & NS \\
\hline \multirow[t]{4}{*}{$31-34$} & \multirow{4}{*}{$\begin{array}{l}\text { Morritt } \\
\text { et al. [6] }\end{array}$} & \multirow[t]{4}{*}{2012} & $48, \mathrm{M}$ & Chin & NS & 1.5 & 1.5 & Excision & No recurrence & NS \\
\hline & & & $68, \mathrm{M}$ & Back & NS & 72 & NS & Excision & No recurrence & NS \\
\hline & & & $72, \mathrm{~F}$ & Scalp & NS & 240 & 24 & Excision & No recurrence & NS \\
\hline & & & $60, \mathrm{~F}$ & Cheek & NS & 3 & NS & Excision & No recurrence & NS \\
\hline 35 & $\begin{array}{l}\text { Skroza } \\
\text { et al. [7] }\end{array}$ & 2014 & $63, \mathrm{M}$ & Scalp & 3 & 24 & NS & Excision & Recurrence & NS \\
\hline 36 & $\begin{array}{l}\text { Sridevi } \\
\text { et al. [8] }\end{array}$ & 2015 & 68, M & R neck & 5.7 & 12 & 3 & Partial excision & NS & NS \\
\hline 37 & $\begin{array}{l}\text { Sakamoto } \\
\text { et al. [9] }\end{array}$ & 2015 & $41, \mathrm{M}$ & $\mathrm{R}$ thumb & 2 & 0.5 & 0.5 & $\begin{array}{l}\text { Excision, } \\
\text { amputation }\end{array}$ & No recurrence & NS \\
\hline 38 & $\begin{array}{l}\text { Suhani } \\
\text { et al. [10] }\end{array}$ & 2015 & $60, \mathrm{~F}$ & $\mathrm{R}$ breast & 5 & 6 & NS & $\begin{array}{l}\text { Modified radical } \\
\text { mastectomy }\end{array}$ & No recurrence & NS \\
\hline 39 & $\begin{array}{l}\text { Lee et al. } \\
{[11]}\end{array}$ & 2016 & $62, \mathrm{M}$ & L cheek & 2 & 3 & 3 & Excision & No recurrence & NS \\
\hline 40 & $\begin{array}{l}\text { Sze et al. } \\
{[12]}\end{array}$ & 2016 & $65, \mathrm{~F}$ & R labia & 5 & 480 & 1.5 & Excision & Residual lesion & NS \\
\hline 41 & $\begin{array}{l}\text { Mohan } \\
\text { et al. [13] }\end{array}$ & 2016 & 28, M & L neck & 6.5 & 7 & 7 & Excision & NS & $\begin{array}{l}\text { "Cut } \\
\text { section" }\end{array}$ \\
\hline 42 & $\begin{array}{l}\text { Current } \\
\text { case }\end{array}$ & 2017 & $64, \mathrm{~F}$ & Neck & 2.1 & 48 & 12 & Excision & No recurrence & Bisection \\
\hline
\end{tabular}

NS, not stated; L, left; R, right; RT, radiation therapy; CT, chemotherapy; Mets, metastasis. ${ }^{1}$ Maximum cyst diameter. ${ }^{2}$ Total duration of cyst presence. ${ }^{3}$ Duration of new signs and symptoms (s/s) warranting evaluation. ${ }^{4}$ Sectioning technique used for histological examination. 